\title{
INSTABILITY OF PERIODIC BGK WAVES
}

\author{
ZHIWU LiN
}

\begin{abstract}
We investigate the stability of periodic Bernstein-Greene-Kruskal (BGK) waves. It is proved that any such wave is linearly unstable under some perturbation of twice the period. A new method is developed to find a growing mode.
\end{abstract}

\section{Introduction}

We consider a one-dimensional electron plasma with a fixed homogeneous neutralizing ion background. In such a plasma collisions are relatively rare; hence we assume no collisions at all. So the time evolution can be modeled by the Vlasov-Poisson system

$$
\begin{gathered}
\frac{\partial f}{\partial t}+v \frac{\partial f}{\partial x}-E \frac{\partial f}{\partial v}=0 \\
\frac{\partial E}{\partial x}=-\int_{-\infty}^{+\infty} f d v+1,
\end{gathered}
$$

where $f(x, v, t)$ is the electron distribution function, $E(x, t)$ the electric field, and 1 is the ion density. In 1957, Bernstein, Greene and Kruskal ([1]) showed the existence of an infinite family of exact stationary solutions to (1), called BGK waves. Since then, the stability of these BGK waves has been of great interest. For example, in [2], [3], [4], [5], numerical computations and formal analysis are used to study the instability and long time behavior. In a series of recent papers ([10],[11],[12],[13],[14]), Guo and Strauss initiated a rigorous study of instability of spatially-dependent equilibria in plasmas. Using some delicate perturbation arguments, they proved in particular that slightly inhomogeneous periodic BGK waves are unstable, if the corresponding homogeneous case has some growing mode according to Penrose's criteria.

In this paper, we consider an arbitrary periodic BGK wave. We prove that it must be linearly unstable. The novelty is that the unperturbed BGK wave, which is spatially dependent, may have an arbitrarily large electric field. Thus we rigorously justify for the first time what was conjectured by physicists ([3], [4], [5], [6]).

Received April 24, 2001. 
A BGK wave is a steady state of the form

$$
f_{0}(x, v)=\mu\left(\frac{1}{2} v^{2}-\beta(x)\right), E_{0}(x)=-\beta_{x}(x),
$$

with $\beta$ a periodic solution of the differential equation

$$
\beta_{x x}=\int_{-\infty}^{+\infty} \mu\left(\frac{1}{2} v^{2}-\beta(x)\right) d v-1 .
$$

We assume that (i) $\mu(\theta)$ is a nonnegative $C^{1}$ function on $\mathbf{R}$; (ii) $\mu$ is neutral, that is,

$$
\int_{-\infty}^{+\infty} \mu\left(\frac{1}{2} v^{2}\right) d v-1=0
$$

and (iii) $\mu$ decays at infinity, namely, for some $\gamma>1$

$$
\left|\mu^{\prime}(\theta)\right| \leq \frac{C}{1+|\theta|^{\gamma}}
$$

Let $\beta$ be any periodic solution of (2). Let $P_{\beta}$ be its minimal period . By adjusting the starting point, we can arrange that the solution satisfies:

$$
\begin{gathered}
\beta(0)=\beta\left(P_{\beta}\right)=\min _{0 \leq x \leq P_{\beta}} \beta(x), \quad \beta\left(\frac{P_{\beta}}{2}\right)=\max _{0 \leq x \leq P_{\beta}} \beta(x), \\
\beta(x)=\beta\left(P_{\beta}-x\right), \quad \forall x \in\left[0, P_{\beta}\right],
\end{gathered}
$$

and $\beta(x)$ is strictly increasing in $\left[0, \frac{P_{\beta}}{2}\right]$.

Our main theorem is

Theorem 1. Let $\left(E_{0}, f_{0}\right)$ be the above periodic BGK solution to (1) with period $P_{\beta}$. Then there exists a $2 P_{\beta}$-periodic growing mode for the linearized equation. That is, there is a solution $\left(e^{\lambda t} \tilde{f}, e^{\lambda t} \tilde{E}\right)$ with $\lambda>0$ to

$$
\begin{gathered}
\frac{\partial f}{\partial t}+v \frac{\partial f}{\partial x}-E_{0} \frac{\partial f}{\partial v}=E \frac{\partial f_{0}}{\partial v} \\
\frac{\partial E}{\partial x}=-\int_{-\infty}^{+\infty} f d v
\end{gathered}
$$

where $\tilde{f}$ and $\tilde{E}$ are $2 P_{\beta}$-periodic functions in $x$ and $\tilde{E} \in H_{l o c}^{1}$.

Now we sketch the main idea in the proof. First, we write the usual dispersion relation in terms of a family of operators $A_{\lambda}$, with $\lambda$ positive denoting the possible growing rate. The key observation is that $A_{\lambda}$ is self-adjoint and is a relatively compact perturbation of $-\partial^{2} / \partial x^{2}$. To show the existence of a growing mode, it is sufficient to find some $\lambda>0$ such that $A_{\lambda}$ has nontrivial kernel. For this purpose, we study the limiting behavior of $A_{\lambda}$, as $\lambda \rightarrow 0$ and $\lambda \rightarrow+\infty$. It turns out that $\lim _{\lambda \rightarrow+\infty} A_{\lambda}$ is nonnegative. So it suffices to prove that $\lim _{\lambda \rightarrow 0} A_{\lambda}$ has some negative subspace. In the multi-periodic case, permitting $k P_{\beta}$ periodicity 
of the perturbation for some integer $k \geq 2$, we can indeed construct such negative function explicitly, using a symmetry property of the problem. Thus we prove the instability for multi-periodic case. The same method can be used to treat the relativistic case. The same conclusion holds, that is, periodic BGK waves are always unstable under multi-periodic perturbations.

In the exact one-periodic case, the situation is more complicated. In general, we can not hope that all periodic BGK waves are unstable under $P_{\beta}$-periodic perturbations. But it is already known that some weakly inhomogeneous BGK waves are unstable (see [11], [6]) under such perturbations. Using our method, we can recover these results as well.

This paper is organized as follows. In section 2, we define the family of dispersion operators. Then we study its properties and limiting behavior. In section 3 , we prove the instability under $k P_{\beta}$-periodic perturbations.

\section{Dispersion operator}

In this section, we define a family of operators to study the eigenvalue problem of (5). Then we study some properties of these operators, especially their limiting behavior. This behavior enables us to get the instability results in the later sections.

For $\lambda>0$, the dispersion operators are defined as

$$
A_{\lambda} \phi:=-\frac{\partial^{2}}{\partial x^{2}} \phi-\int_{\mathbf{R}} \mu^{\prime}(e) d v \phi(x)+\int_{\mathbf{R}} \mu^{\prime}(e) \int_{-\infty}^{0} \lambda e^{\lambda s} \phi(\mathbf{X}(s)) d s d v,
$$

where $e=\frac{1}{2} v^{2}-\beta(x)$, and $(\mathbf{X}(s ; x, v), \mathbf{V}(s ; x, v))$ is the solution of the characteristic equation

$$
\begin{gathered}
\dot{\mathbf{X}}(s)=\mathbf{V}(s), \\
\dot{\mathbf{V}}(s)=-E_{0}(\mathbf{X}(s)),
\end{gathered}
$$

with initial data $\mathbf{X}(0)=x, \mathbf{V}(0)=v$. Note that $e=\frac{1}{2} \mathbf{V}(s)^{2}-\beta(\mathbf{X}(s))$ is independent of $s$. Note that $A_{\lambda}: \mathcal{H} \rightarrow \mathcal{L}$, where $\mathcal{L}$ is the space of $P_{\beta}$-periodic functions with zero average that are $L^{2}$ over a period $P=k P_{\beta}$, and $\mathcal{H}$ is the subspace of functions that are $H^{2}$ over the same period.

Lemma 2. $A_{\lambda}$ is well-defined by our assumption on $\mu$. Indeed, if denote

$$
K_{\lambda} \phi:=-\int_{\mathbf{R}} \mu^{\prime}(e) d v \phi(x)+\int_{\mathbf{R}} \mu^{\prime}(e) \int_{-\infty}^{0} \lambda e^{\lambda s} \phi(\mathbf{X}(s)) d s d v,
$$

then we have

$$
\left\|K_{\lambda} \phi\right\|_{2} \leq 2 \sup _{x \in[0, P]} \int_{\mathbf{R}}\left|\mu^{\prime}(e)\right| d v\|\phi\|_{2} .
$$


Proof. Take any $\psi \in L^{2}(0, P)$, then

$$
\begin{aligned}
& \left|\int_{0}^{P} \int_{\mathbf{R}} \int_{-\infty}^{0} \lambda e^{\lambda s} \mu^{\prime}(e) \phi(\mathbf{X}(s)) \psi(x) d s d v d x\right| \\
& \leq\left(\int_{0}^{P} \int_{\mathbf{R}} \int_{-\infty}^{0} \lambda e^{\lambda s}|\phi|^{2}\left|\mu^{\prime}(e)\right|(\mathbf{X}(s), \mathbf{V}(s)) d s d v d x\right)^{\frac{1}{2}} . \\
& \left(\int_{0}^{P} \int_{\mathbf{R}} \int_{-\infty}^{0} \lambda e^{\lambda s}|\psi|^{2}\left|\mu^{\prime}(e)\right| d s d v d x\right)^{\frac{1}{2}} \\
& \leq\left(\int_{-\infty}^{0} \lambda e^{\lambda s} \sup _{x \in[0, P]} \int_{\mathbf{R}}\left|\mu^{\prime}(e)\right| d v \int_{0}^{P}|\phi|^{2} d x d s\right)^{\frac{1}{2}} . \\
& \left(\int_{-\infty}^{0} \lambda e^{\lambda s} \sup _{x \in[0, P]} \int_{\mathbf{R}}\left|\mu^{\prime}(e)\right| d v \int_{0}^{P}|\psi|^{2} d x d s\right)^{\frac{1}{2}} \\
& =\sup _{x \in[0, P]} \int_{\mathbf{R}} \mu^{\prime}(e) \mid d v\|\phi\|_{2}\|\psi\|_{2} .
\end{aligned}
$$

Here in the first integral of second inequality, we made the change of variable $(x, v) \rightarrow(\mathbf{X}(s), \mathbf{V}(s))$. So we have

$$
\left\|\int_{\mathbf{R}} \mu^{\prime}(e) \int_{-\infty}^{0} \lambda e^{\lambda s} \phi(\mathbf{X}(s)) d s d v\right\|_{2} \leq \sup _{x \in[0, P]} \int_{\mathbf{R}}\left|\mu^{\prime}(e)\right| d v\|\phi\|_{2}
$$

and the conclusion follows.

The following lemma indicates the reason why we introduce $A_{\lambda}$.

Lemma 3. Let $\lambda>0$. Then there exists some nontrivial solution $\left(e^{\lambda t} f, e^{\lambda t} E\right)$ to (5) with $f \in L^{\infty} \cap C^{1}$ and $E \in H^{1}$, if and only if there exists some non-constant $\phi \in \mathcal{H}$, such that $A_{\lambda} \phi=0$. In this case,

$$
f(x, v)=-\mu^{\prime}(e) \phi(x)+\mu^{\prime}(e) \int_{-\infty}^{0} \lambda e^{\lambda s} \phi(\mathbf{X}(s ; x, v)) d s
$$

and $E=-\partial_{x} \phi$.

Proof. If $\left(e^{\lambda t} f, e^{\lambda t} E\right)$ is a solution to (5) with $f \in L^{\infty}, E \in H^{1}$ and $E=$ $-\partial_{x} \phi$, then $\phi \in \mathcal{H}$. From the Vlasov equation(5a) we get

$$
\lambda f+v \partial_{x} f-E_{0} \partial_{v} f=-\partial_{x} \phi v \mu^{\prime}(e) .
$$

By (9) we have

$$
\frac{d}{d s}\left(e^{\lambda s} f(\mathbf{X}(s), \mathbf{V}(s))\right)=-e^{\lambda s} \phi^{\prime}(\mathbf{X}(s)) \mathbf{V}(s) \mu^{\prime}(e) .
$$


Integrating (10) from $-\infty$ to 0 , we get

$$
\begin{aligned}
f(x, v) & =-\int_{-\infty}^{0} e^{\lambda s} \mu^{\prime}(e) \phi^{\prime}(\mathbf{X}(s)) \mathbf{V}(s) d s \\
& =-\int_{-\infty}^{0} e^{\lambda s} \mu^{\prime}(e) \frac{d}{d s}(\phi(\mathbf{X}(s))) d s \\
& =-\mu^{\prime}(e) \phi(x)+\mu^{\prime}(e) \int_{-\infty}^{0} \lambda e^{\lambda s} \phi(\mathbf{X}(s)) d s
\end{aligned}
$$

Placing this expression into the Poisson equation( $5 b)$, we get

$$
\frac{\partial^{2} \phi}{\partial x^{2}}=-\int_{\mathbf{R}} \mu^{\prime}(e) d v \phi(x)+\int_{\mathbf{R}} \mu^{\prime}(e) \int_{-\infty}^{0} \lambda e^{\lambda s} \phi(\mathbf{X}(s)) d s d v
$$

which is just $A_{\lambda} \phi=0$.

Conversely, if there exists some $\phi \in \mathcal{H}$ such that $A_{\lambda} \phi=0$, then $\frac{\partial E}{\partial x}=$ $-\int_{-\infty}^{+\infty} f d v$ is automatically satisfied for $E=-\partial_{x} \phi$ and $f$ determined by (8). We only need to check $(9)$.

Setting $(x, v)$ to be $(\mathbf{X}(t), \mathbf{V}(t))$ in $(8)$, we get

$$
\begin{aligned}
f(\mathbf{X}(t), \mathbf{V}(t)) & =-\mu^{\prime}(e) \phi(\mathbf{X}(t))+\mu^{\prime}(e) \int_{-\infty}^{0} \lambda e^{\lambda s} \phi(\mathbf{X}(s ; 0, \mathbf{X}(t), \mathbf{V}(t))) d s \\
& =-\mu^{\prime}(e) \phi(\mathbf{X}(t))+\mu^{\prime}(e) \int_{-\infty}^{0} \lambda e^{\lambda s} \phi(\mathbf{X}(s+t)) d s \\
& =-\mu^{\prime}(e) \phi(\mathbf{X}(t))+\mu^{\prime}(e) e^{-\lambda t} \int_{-\infty}^{t} \frac{d}{d s}\left(e^{\lambda s}\right) \phi(\mathbf{X}(s)) d s \\
& =-\mu^{\prime}(e) \phi(\mathbf{X}(t))+\mu^{\prime}(e) e^{-\lambda t} \\
& \left(e^{\lambda t} \phi(\mathbf{X}(t))-\int_{-\infty}^{t} e^{\lambda s} \phi^{\prime}(\mathbf{X}(s)) \mathbf{V}(s) d s\right) \\
& =-\mu^{\prime}(e) e^{-\lambda t} \int_{-\infty}^{t} e^{\lambda s} \phi^{\prime}(\mathbf{X}(s)) \mathbf{V}(s) d s
\end{aligned}
$$

So

$$
e^{\lambda t} f(\mathbf{X}(t), \mathbf{V}(t))=-\mu^{\prime}(e) \int_{-\infty}^{t} e^{\lambda s} \phi^{\prime}(\mathbf{X}(s)) \mathbf{V}(s) d s .
$$

Differentiating both sides, we get

$$
\frac{d}{d t}\left(e^{\lambda t} f(\mathbf{X}(t), \mathbf{V}(t))\right)=-e^{\lambda t} \phi^{\prime}(\mathbf{X}(t)) \mathbf{V}(t) \mu^{\prime}(e)
$$

which is equivalent to (9). 
Next we study some properties of $A_{\lambda}$.

Lemma 4. $A_{\lambda}$ is self-adjoint on $\mathcal{L}$ and has purely discrete spectrum.

Proof. We write

$$
A_{\lambda}=--\frac{\partial^{2}}{\partial x^{2}}+K_{\lambda}
$$

where $K_{\lambda}$ is defined by (6).

First we show that $K_{\lambda}$ is symmetric. For any $\phi, \psi \in L^{2}(0, P)$, we have

$$
\begin{aligned}
& \int_{0}^{P} \int_{\mathbf{R}} \int_{-\infty}^{0} \lambda e^{\lambda s} \phi(\mathbf{X}(s)) \mu^{\prime}(e) \psi(x) d s d v d x \\
= & \int_{0}^{P} \int_{\mathbf{R}} \int_{-\infty}^{0} \lambda e^{\lambda s} \phi(x) \mu^{\prime}(e) \psi(\mathbf{X}(-s)) d s d v d x \\
= & \int_{0}^{P} \int_{\mathbf{R}} \int_{-\infty}^{0} \lambda e^{\lambda s} \phi(x) \mu^{\prime}(e) \psi(\mathbf{X}(s)) d s d v d x .
\end{aligned}
$$

So $\left(K_{\lambda} \phi, \psi\right)=\left(K_{\lambda} \psi, \phi\right)$ follows. Here in the second equality, we made the change of variable $(x, v) \rightarrow(\mathbf{X}(s), \mathbf{V}(s))$. In the third line, we changed $v$ to $-v$ and used the facts that

$$
(\mathbf{X}(s ; x, v), \mathbf{V}(s ; x, v))=(\mathbf{X}(-s ; x,-v),-\mathbf{V}(-s ; x,-v))
$$

and $e=\frac{1}{2} v^{2}-\phi(x)$ is an invariant along the characteristic curve.

Next we claim that $K_{\lambda}$ is a relatively compact perturbation of $-\frac{\partial^{2}}{\partial x^{2}}$. For convenience, we recall the definition of relative compactness from [17]. Let $A$ be selfadjoint. An operator $C$ with $D(A) \subset D(C)$ is called relatively compact with respect to $A$ if and only if $C$ is compact restricted to the space $\left(D(A),\|\cdot\|_{A}\right)$. Here $\|\phi\|_{A}^{2}=\|\phi\|^{2}+\|A \phi\|^{2}$. Then in our case, we need to show that $K_{\lambda}$ is compact from $H^{2}$ to $L^{2}$. By Lemma $2, K_{\lambda}$ is a bounded operator from $L^{2}$ to $L^{2}$. Combining it with the fact that the inclusion $H^{2} \hookrightarrow L^{2}$ is compact, we get the compactness of $K_{\lambda}: H^{2} \rightarrow L^{2}$.

Thus by the Kato-Rellich Theorem(see [17]), $A_{\lambda}$ is self-adjoint. By Weyl's Theorem for self-adjoint operators $($ see $[17]), \sigma_{\text {ess }} \quad\left(A_{\lambda}\right)=\sigma_{\text {ess }} \quad\left(-\frac{\partial^{2}}{\partial x^{2}}\right)$. Since we know that $-\frac{\partial^{2}}{\partial x^{2}}$ with periodic boundary condition has only discrete spectrum, $A_{\lambda}$ also has only discrete spectrum.

The operator $A_{\lambda}$ is quite complicated since it depends on the particle orbits. In the following, we will study the limiting behavior of $A_{\lambda}$ when $\lambda$ is near 0 or $+\infty$. For our later purpose, we only need to study the following problem:

If $\phi \in H^{1}(0, P)$, what is the limit of $\left(A_{\lambda} \phi, \phi\right)$ as $\lambda \rightarrow 0$ and $\lambda \rightarrow+\infty$ ? 
2.1. Limiting behavior near $\lambda=0$. First we prove a simple fact which will be used later.

Lemma 5. For any T-periodic function $\phi(x) \in L^{1}(0, T)$, we have

$$
\lim _{\lambda \rightarrow 0^{+}} \int_{-\infty}^{0} e^{s} \phi\left(\frac{s}{\lambda}\right) d s=\frac{1}{T} \int_{0}^{T} \phi(s) d s .
$$

Proof.

$$
\begin{aligned}
\int_{-\infty}^{0} e^{s} \phi\left(\frac{s}{\lambda}\right) d s & =\int_{0}^{+\infty} e^{-s} \phi\left(\frac{-s}{\lambda}\right) d s \\
& =\sum_{k=0}^{\infty} \int_{k \lambda T}^{(k+1) \lambda T} e^{-s} \phi\left(\frac{-s}{\lambda}\right) d s \\
& =\sum_{k=0}^{\infty} e^{-k \lambda T} \int_{0}^{\lambda T} e^{-s} \phi\left(\frac{-s}{\lambda}\right) d s \\
& =\frac{\lambda}{1-e^{-\lambda T}} \int_{0}^{T} e^{-\lambda s} \phi(-s) d s
\end{aligned}
$$

So

$$
\begin{aligned}
\lim _{\lambda \rightarrow 0^{+}} \int_{-\infty}^{0} e^{s} \phi\left(\frac{s}{\lambda}\right) d s & =\lim _{\lambda \rightarrow 0^{+}} \frac{\lambda}{1-e^{-\lambda T}} \int_{0}^{T} e^{-\lambda s} \phi(-s) d s \\
& =\frac{1}{T} \int_{0}^{T} \phi(-s) d s \\
& =\frac{1}{T} \int_{0}^{T} \phi(s) d s .
\end{aligned}
$$

Now we study the solutions to the characteristic equation

$$
\begin{gathered}
\dot{\mathbf{X}}(s)=\mathbf{V}(s), \\
\dot{\mathbf{V}}(s)=-E_{0}(\mathbf{X}(s)),
\end{gathered}
$$

with initial conditions $\mathbf{X}(0)=x, \mathbf{V}(0)=v$. The energy $e=\frac{1}{2} v^{2}-\beta(x)$ is an invariant. According to the size of $e$, we can divide the particles into two types:

1. The free particles have energy $e>-\min \beta(x)$. They travel through the whole interval $[0, P]$, then go back and forth. The period is

$$
P_{f}=\int_{0}^{P_{\beta}} \frac{1}{\sqrt{2(e+\beta(y))}} d y .
$$

2. The trapped particles have energy $-\max \beta(x) \leq e \leq-\min \beta(x)$.Let $k \in \mathbb{N}$ and let $P=k P_{\beta}$. According to the properties of our BGK solution (stated in the introduction), there exists an unique interval $(a, b)$ in $\left[0, P_{\beta}\right]$, such that $e+\beta(a)=e+\beta(b)=0$, and $e+\beta(x)>0$ for $x \in(a, b)$. Thus the particles are trapped in exactly one of the $k$ intervals $[a, b],\left[a+P_{\beta}, b+P_{\beta}\right], \cdots,[a+$ 
$\left.(k-1) P_{\beta}, b+(k-1) P_{\beta}\right]$. Denoting $a_{i}=a+(i-1) P_{\beta}, b_{i}=b+(i-1) P_{\beta}$, the half period of the particle trapped in $\left[a_{i}, b_{i}\right]$ is

$$
P_{t}=\int_{a_{i}}^{b_{i}} \frac{1}{\sqrt{2(e+\beta(y))}} d y=\int_{a}^{b} \frac{1}{\sqrt{2(e+\beta(y))}} d y .
$$

Now we can state the result concerning the limit as $\lambda \rightarrow 0$.

Lemma 6. For any $\phi \in H^{1}(0, P)$, we have

$$
\begin{aligned}
\lim _{\lambda \rightarrow 0^{+}}\left(A_{\lambda} \phi, \phi\right) & =\int_{0}^{P}\left\{\left|\phi_{x}\right|^{2}-\int_{\mathbf{R}} \mu^{\prime}(e) d v|\phi|^{2}\right\} d x \\
& +\int_{-\min \beta(x)}^{\infty} \mu^{\prime}(e) \frac{1}{P_{f}}\left(\int_{0}^{P} \frac{\phi(y)}{\sqrt{2(e+\beta(y))}} d y\right)^{2} d e \\
& +2 \int_{-\max \beta(x)}^{-\min \beta(x)} \mu^{\prime}(e) \frac{1}{P_{t}} \sum_{i=1}^{k}\left(\int_{a_{i}}^{b_{i}} \frac{\phi(y)}{\sqrt{2(e+\beta(y))}} d y\right)^{2} d e .
\end{aligned}
$$

We denote this limit by $a_{0}(\phi)$.

Proof. First we note that for any particle orbit with initial $(x, v)$, by Lemma 5 the following limit holds

$$
\begin{aligned}
\lim _{\lambda \rightarrow 0^{+}} \int_{-\infty}^{0} \lambda e^{\lambda s} \phi(\mathbf{X}(s)) d s & =\lim _{\lambda \rightarrow 0^{+}} \int_{-\infty}^{0} e^{s} \phi\left(\mathbf{X}\left(\frac{s}{\lambda}\right)\right) d s \\
& =\left\{\begin{array}{cc}
\frac{1}{2 P_{f}} \int_{0}^{2 P_{f}} \phi(\mathbf{X}(s)) d s & \text { if } e>-\min \beta \\
\frac{1}{2 P_{t}} \int_{0}^{2 P_{t}} \phi(\mathbf{X}(s)) d s & \text { if }-\max \beta \leq e \leq-\min \beta
\end{array}\right. \\
& =\left\{\begin{array}{cc}
\frac{1}{2 P_{f}} \int_{0}^{P} \frac{\phi(y)}{\sqrt{2(e+\beta(y))}} d y & \text { if } e>-\min \beta \\
\frac{1}{P_{t}} \int_{a_{i}}^{b_{i}} \frac{\phi(y)}{\sqrt{2(e+\beta(y))}} d y & \text { if }-\max \beta \leq e \leq-\min \beta
\end{array}\right.
\end{aligned}
$$

Here in the third line, we change of variable $s$ to $y=\mathbf{X}(s)$, and notice that $\frac{d \mathbf{X}(s)}{d s}=\mathbf{V}(s)=\sqrt{2(e+\beta(\mathbf{X}(s)))}$. By definition,

$$
\begin{aligned}
\left(A_{\lambda} \phi, \phi\right)= & \int_{0}^{P}\left\{\left|\phi_{x}\right|^{2}-\int_{\mathbf{R}} \mu^{\prime}(e) d v|\phi|^{2}\right\} d x+ \\
& \int_{0}^{P} \int_{\mathbf{R}} \mu^{\prime}(e) \phi(x) \int_{-\infty}^{0} \lambda e^{\lambda s} \phi(\mathbf{X}(s)) d s d v d x .
\end{aligned}
$$


In the last term, the integrand is $\leq \lambda\left|\mu^{\prime}(e)\right||\phi(x)||\phi|_{L^{\infty}}$, which is integrable. So by Dominated Convergence Theorem, we have

$$
\begin{aligned}
& \lim _{\lambda \rightarrow 0^{+}} \int_{0}^{P} \int_{\mathbf{R}} \mu^{\prime}(e) \phi(x) \int_{-\infty}^{0} \lambda e^{\lambda s} \phi(\mathbf{X}(s)) d s d v d x \\
& =2 \int_{-\min \beta}^{\infty} \mu^{\prime}(e) \int_{0}^{P} \frac{\phi(x)}{\sqrt{2(e+\beta(x))}} \lim _{\lambda \rightarrow 0^{+}} \int_{-\infty}^{0} \lambda e^{\lambda s} \phi(\mathbf{X}(s)) d s d x d e \\
& +2 \int_{-\max \beta}^{-\min \beta} \mu^{\prime}(e) \sum_{i=1}^{k} \int_{a_{i}}^{b_{i}} \frac{\phi(x)}{\sqrt{2(e+\beta(x))}} \lim _{\lambda \rightarrow 0^{+}} \int_{-\infty}^{0} \lambda e^{\lambda s} \phi(\mathbf{X}(s)) d s d x d e \\
& =\int_{-\min \beta}^{\infty} \mu^{\prime}(e) \frac{1}{P_{f}}\left(\int_{0}^{P} \frac{\phi(y)}{\sqrt{2(e+\beta(y))}}\right)^{2} d e \\
& +2 \int_{-\max \beta}^{-\min \beta} \mu^{\prime}(e) \frac{1}{P_{t}} \sum_{i=1}^{k}\left(\int_{a_{i}}^{b_{i}} \frac{\phi(y)}{\sqrt{2(e+\beta(y))}}\right)^{2} d e .
\end{aligned}
$$

Here in the second line, we changed variables $v \rightarrow e$, and changed the order of the integrals of $e, x$. Thus we complete the proof of the lemma.

2.2. Limiting behavior near $\lambda=+\infty$. Next we show that when $\lambda \rightarrow+\infty$, $A_{\lambda}$ is nonnegative.

Lemma 7. There exists $\Lambda_{0}>0$ such that if $\lambda>\Lambda_{0}$ then $A_{\lambda}$ is nonnegative.

Proof. First we claim that: if $\phi \in H^{1}(0, P)$, then $\left\|K_{\lambda} \phi\right\|_{2} \leq \frac{c}{\lambda}\left\|\phi_{x}\right\|_{2}$.

To prove the claim, let $\psi \in L^{2}(0, P)$. Let $\alpha=1-\varepsilon-\frac{3}{4 \gamma}$, where $\varepsilon$ is small. Then

$$
\begin{aligned}
\left|\left(K_{\lambda} \phi, \psi\right)\right| & =\left|\int_{0}^{P} \int_{\mathbf{R}} \int_{-\infty}^{0} e^{\lambda s} \mu^{\prime}(e) \phi^{\prime}(\mathbf{X}(s)) \mathbf{V}(s) \psi(x) d s d v d x\right| \\
& \leq\left(\int_{0}^{P} \int_{\mathbf{R}} \int_{-\infty}^{0} e^{\lambda s}\left|\phi^{\prime}(\mathbf{X})\right|^{2}\left|\mu^{\prime}\left(\frac{1}{2} \mathbf{V}^{2}-\beta(\mathbf{X})\right)\right|^{2(1-\alpha)} \mathbf{V}(s)^{2} d s d v d x\right)^{\frac{1}{2}} . \\
& \left(\int_{0}^{P} \int_{\mathbf{R}} \int_{-\infty}^{0} e^{\lambda s}|\psi|^{2}\left|\mu^{\prime}(e)\right|^{2 \alpha} d s d v d x\right)^{\frac{1}{2}} \\
& \leq\left(\int_{-\infty}^{0} e^{\lambda s} d s \sup _{\mathbf{X} \in[0, P]} \int_{\mathbf{R}} \mathbf{V}^{2}\left|\mu^{\prime}(e)\right|^{2(1-\alpha)} d \mathbf{V} \int_{0}^{P}\left|\phi^{\prime}(\mathbf{X})\right|^{2} d \mathbf{X}\right)^{\frac{1}{2}} . \\
& \left(\int_{-\infty}^{0} e^{\lambda s} \sup _{x \in[0, P]} \int_{\mathbf{R}}\left|\mu^{\prime}(e)\right|^{2 \alpha} d v \int_{0}^{P}|\psi|^{2} d x d s\right)^{\frac{1}{2}} \\
& =\frac{c}{\lambda}\left\|\phi^{\prime}\right\|_{2}\|\psi\|_{2} .
\end{aligned}
$$


Thus $\left\|K_{\lambda} \phi\right\|_{2} \leq \frac{c}{\lambda}\left\|\phi_{x}\right\|_{2}$. Here in the second line, we changed variables $(\mathbf{X}(s), \mathbf{V}(s))$ to $(x, v)$ since the Jacobian $=1$. From the definition of $\alpha$ and $\gamma>1$, it is easy to check that $4 \alpha \gamma>1$ and $4(1-\alpha) \gamma-2>1$. Thus by the assumption on $\mu$, we have

$$
c=\left(\sup _{x \in[0, P]} \int_{\mathbf{R}} v^{2}\left|\mu^{\prime}(e)\right|^{2(1-\alpha)} d v \cdot \sup _{x \in[0, P]} \int_{\mathbf{R}}\left|\mu^{\prime}(e)\right|^{2 \alpha} d v\right)^{\frac{1}{2}}<\infty .
$$

Suppose now that there exists some $\phi \neq 0 \in \mathcal{H}, k<0$ such that $A_{\lambda} \phi=k \phi$. That is,

$$
-\frac{\partial^{2}}{\partial x^{2}} \phi-\int \mu^{\prime}(e) d v \phi(x)+\int_{\mathbf{R}} \mu^{\prime}(e) \int_{-\infty}^{0} \lambda e^{\lambda s} \phi(\mathbf{X}(s)) d s d v=k \phi .
$$

Integrating both sides, we get

$$
\begin{aligned}
k \int_{0}^{P} \phi(x) d x & =-\int_{0}^{P} \int_{\mathbf{R}} \mu^{\prime}(e) \phi(x) d v d x \\
& +\int_{0}^{P} \int_{\mathbf{R}} \int_{-\infty}^{0} \lambda e^{\lambda s} \phi(\mathbf{X}(s)) \mu^{\prime}(e) d s d v d x \\
& =-\int_{0}^{P} \int_{\mathbf{R}} \mu^{\prime}(e) \phi(x) d v d x \\
& +\int_{-\infty}^{0} \lambda e^{\lambda s} \int_{0}^{P} \int_{\mathbf{R}} \mu^{\prime}(e) \phi(\mathbf{X}) d \mathbf{V} d \mathbf{X} d s \\
& =0
\end{aligned}
$$

So $\int_{0}^{P} \phi(x) d x=0$, and thus by Wirtinger's inequality, $\|\phi\|_{2} \leq \frac{P}{2 \pi}\left\|\phi_{x}\right\|_{2}$.

Hence

$$
\begin{aligned}
k(\phi, \phi) & =\left(A_{\lambda} \phi, \phi\right)=\left\|\phi_{x}\right\|_{2}^{2}+\left(K_{\lambda} \phi, \phi\right) \\
& \geq\left\|\phi_{x}\right\|_{2}^{2}-\left\|K_{\lambda} \phi\right\|_{2}\|\phi\|_{2} \\
& \geq\left\|\phi_{x}\right\|_{2}^{2}-\frac{c}{\lambda} \frac{P}{2 \pi}\left\|\phi_{x}\right\|_{2}^{2}>0 .
\end{aligned}
$$

If $\lambda>\Lambda_{0}=\frac{c P}{2 \pi}$. This is a contradiction. Thus $A_{\lambda}$ is nonnegative if $\lambda>\Lambda_{0}$.

We have the following result.

Lemma 8. In case there exists some $\phi_{-} \in H^{1}(0, P)$, such that $a_{0}\left(\phi_{-}\right)<$ 0 (defined in Lemma 6), then there exists $\lambda_{0}>0$, and $\phi_{\lambda_{0}} \neq 0 \in \mathcal{H}$, $\int_{0}^{P} \phi_{\lambda_{0}}(x) d x=0$, such that $\left[e^{\lambda_{0} t} f, e^{\lambda_{0} t} E\right]$ is the solution to linearized problem (5), where

$$
E=-\partial_{x} \phi_{\lambda_{0}}, \quad f(x, v)=-\mu^{\prime}(e) \phi_{\lambda_{0}}(x)+\mu^{\prime}(e) \int_{-\infty}^{0} \lambda e^{\lambda s} \phi_{\lambda_{0}}(\mathbf{X}(s)) d s d v
$$


Proof. By Lemma 1, the conclusion is equivalent to showing that there exists $\lambda_{0}>0$ such that $A_{\lambda_{0}}$ has some nontrivial kernel. By assumption, we know that for some small $\varepsilon_{0}>0, A_{\varepsilon_{0}}$ has a negative eigenvalue. Define $k(\lambda)$ to be the least eigenvalue of $A_{\lambda}(0<\lambda<+\infty)$. We claim that $k(\lambda)$ is a continuous function. To prove this, first we recall the following fact (based on Lemma 4):

$$
k(\lambda)=\inf _{\phi \in D\left(A_{\lambda}\right):\|\phi\|_{2}=1}\left(\phi, A_{\lambda} \phi\right),
$$

where $D\left(A_{\lambda}\right) \equiv \mathcal{H}$. Fixed $\lambda_{0}>0, \phi \in \mathcal{H},\|\phi\|_{2}=1$. Consider $\lambda \in\left(\lambda_{0} / 2,3 \lambda_{0} / 2\right)$. We suppose $\lambda>\lambda_{0}$ in the following. We have

$$
\begin{aligned}
k\left(\lambda_{0}\right) & \leq\left(\phi, A_{\lambda_{0}} \phi\right) \\
& \leq\left(\phi, A_{\lambda} \phi\right)+\left|\left(\phi, A_{\lambda} \phi\right)-\left(\phi, A_{\lambda_{0}} \phi\right)\right| \\
& \leq\left(\phi, A_{\lambda} \phi\right)+\int_{0}^{P} \int_{\mathbf{R}} \int_{-\infty}^{0}\left|\lambda e^{\lambda s}-\lambda_{0} e^{\lambda_{0} s}\right|\left|\phi(x) \mu^{\prime}(e) \phi(\mathbf{X}(s))\right| d s d v d x \\
& \leq\left(\phi, A_{\lambda} \phi\right)+\int_{0}^{P} \int_{\mathbf{R}} \int_{-\infty}^{0} \int_{\lambda_{0}}^{\lambda}\left(\tilde{\lambda}|s| e^{\tilde{\lambda} s}+e^{\tilde{\lambda} s}\right) \\
& \leq\left(\phi, A_{\lambda} \phi\right)+\sup _{x \in[0, P]} \int_{\mathbf{R}}\left|\mu^{\prime}(e)\right| d v \int_{\lambda_{0}}^{\lambda} \int_{-\infty}^{0}\left(\tilde{\lambda}\left|\phi(x) \mu^{\prime}(e) \phi(\mathbf{X}(s))\right| d s d v d x\right. \\
& \left.\leq\left(\phi, A_{\lambda} \phi\right)+\sup _{x \in[0, P]} \int_{\mathbf{R}}\left|\mu^{\prime}(e)\right| d v \int_{\lambda_{0}}^{\lambda} \frac{2}{\tilde{\lambda}} d \tilde{\lambda} s\right) d s d \tilde{\lambda} \\
& \leq\left(\phi, A_{\lambda} \phi\right)+c\left|\ln \lambda-\ln \lambda_{0}\right| .
\end{aligned}
$$

where $c=2 \sup _{x \in[0, P]} \int_{\mathbf{R}}\left|\mu^{\prime}(e)\right| d v$. We can get the same inequality for $\lambda<\lambda_{0}$ in the same way. Here the proof of the fifth inequality is the same as the proof of (7) with $\psi=\phi$. Taking the infimum over all $\phi$ in the above inequality, we get $k\left(\lambda_{0}\right) \leq$ $k(\lambda)+c\left|\ln \lambda-\ln \lambda_{0}\right|$. The same argument gives $k(\lambda) \leq k\left(\lambda_{0}\right)+c\left|\ln \lambda-\ln \lambda_{0}\right|$. Thus we have

$$
\left|k(\lambda)-k\left(\lambda_{0}\right)\right| \leq c\left|\ln \lambda-\ln \lambda_{0}\right|
$$

This proves the continuity of $k(\lambda)$.

Since $k\left(\varepsilon_{0}\right)<0$ by assumption and $k(\lambda) \geq 0$ for $\lambda$ large enough (Lemma 7), there exists $\lambda_{0}>\varepsilon_{0}$ such that $k\left(\lambda_{0}\right)=0$. Thus we get the conclusion.

\section{Instability of multi-periodic BGK waves}

In this section, we prove the main theorem of this paper. That is, we show that $P_{\beta}$-periodic BGK waves are unstable under $k P_{\beta}$-periodic perturbations if $k \geq 2$. By Lemma 8 , we only need to show that there exists some $\phi_{-} \in H^{1}(0, P)$, such that $a_{0}\left(\phi_{-}\right)<0$. Denoting $P=k P_{\beta}(k \geq 2)$, we are able to construct such a test function. This is done in the following lemma. 
Lemma 9. If $P=k P_{\beta}$ for some integer $k \geq 2$, then there exists $\phi_{-} \in H^{1}(0, P)$, such that $a_{0}\left(\phi_{-}\right)<0$.

Proof. We treat the case $k=2$ first.

The BGK waves in Theorem 1 indeed have the following symmetry: $\beta(x)=$ $\beta\left(P_{\beta}-x\right)$, for $x \in\left[0, P_{\beta}\right]$. Denote $\phi_{1}=\partial_{x} \beta$, so that $\phi_{1}(x)=-\phi_{1}\left(P_{\beta}-x\right)$ for $x \in\left[0, P_{\beta}\right]$. The electric potential $\beta$ satisfies the equation:

$$
\frac{\partial^{2}}{\partial x^{2}} \beta=\int_{\mathbf{R}} \mu(e) d v-1
$$

where $e=\frac{1}{2} v^{2}-\beta(x)$. Differentiating the above equation, we get

$$
\frac{\partial^{2}}{\partial x^{2}} \phi_{1}=-\int_{\mathbf{R}} \mu^{\prime}(e) d v \phi_{1}
$$

Note that $\phi_{1}(0)=\phi_{1}\left(P_{\beta}\right)=\phi_{1}\left(\frac{P_{\beta}}{2}\right)=0$. Now consider the following eigenvalue problem:

$$
\frac{\partial^{2}}{\partial x^{2}} \phi+\left(\lambda+\int_{\mathbf{R}} \mu^{\prime}(e) d v\right) \phi=0
$$

where $\phi \in H^{2}\left(\frac{p_{\beta}}{2}, \frac{3 P_{\beta}}{2}\right), \phi\left(\frac{p_{\beta}}{2}\right)=\phi\left(\frac{3 P_{\beta}}{2}\right)=0$. Then by equation (11), $\phi_{1}$ is a solution with $\lambda=0$. Furthermore, $\phi_{1}$ has a zero in $\left(\frac{p_{\beta}}{2}, \frac{3 P_{\beta}}{2}\right)$, so by ODE theory there exists some $\lambda_{-}<0$ and some $\phi_{-} \in H^{2}\left(\frac{p_{\beta}}{2}, \frac{3 P_{\beta}}{2}\right)$ that satisfies $\phi_{-}\left(\frac{p_{\beta}}{2}\right)=\phi_{-}\left(\frac{3 P_{\beta}}{2}\right)=0$ such that

$$
\frac{\partial^{2}}{\partial x^{2}} \phi_{-}+\left(\lambda_{-}+\int_{\mathbf{R}} \mu^{\prime}(e) d v\right) \phi_{-}=0 .
$$

We now extend $\phi_{-}(x)$ to whole interval $\left[0,2 P_{\beta}\right]$ in the following way:

$$
\phi_{-}(x)=\left\{\begin{array}{cc}
-\phi_{-}\left(P_{\beta}-x\right) & \text { if } x \in\left[0, \frac{P_{\beta}}{2}\right] \\
-\phi_{-}\left(3 P_{\beta}-x\right) & \text { if } x \in\left[\frac{3 P_{\beta}}{2}, 2 P_{\beta}\right] .
\end{array}\right.
$$

Then $\phi_{-}(0)=\phi_{-}\left(2 P_{\beta}\right)$, and $\partial_{x} \phi_{-}(0)=\partial_{x} \phi_{-}\left(2 P_{\beta}\right)=\partial_{x} \phi_{-}\left(P_{\beta}\right)$. Thus $\phi_{-}$is a $2 P_{\beta}$-periodic function in $H^{1}\left(0,2 P_{\beta}\right)$. By our construction it is obvious that 


$$
\begin{aligned}
\int_{0}^{2 P_{\beta}}\left\{\left|\partial_{x} \phi_{-}\right|^{2}-\int_{\mathbf{R}} \mu^{\prime}(e) d v\left|\phi_{-}\right|^{2}\right\} & d x \\
& =2 \int_{\frac{p_{\beta}}{2}}^{\frac{3 P_{\beta}}{2}}\left\{\left|\partial_{x} \phi_{-}\right|^{2}-\int_{\mathbf{R}} \mu^{\prime}(e) d v\left|\phi_{-}\right|^{2}\right\} d x \\
& =2 \lambda_{-} \int_{\frac{p_{\beta}}{2}}^{\frac{3 P_{\beta}}{2}}\left|\phi_{-}\right|^{2} d x<0 .
\end{aligned}
$$

Now we compute $a_{0}\left(\phi_{-}\right)$.We claim that:

$$
\int_{0}^{2 P_{\beta}} \frac{\phi_{-}(y)}{\sqrt{2(e+\beta(y))}} d y=0, \quad \int_{a_{i}}^{b_{i}} \frac{\phi_{-}(y)}{\sqrt{2(e+\beta(y))}} d y=0
$$

where the above expressions appear in the definition of $a_{0}(\phi)$ in Lemma 6). In fact, this follows directly from our construction of $\phi_{-}(x)$ and the symmetry of $\beta(x): \beta(x)=\beta\left(P_{\beta}-x\right)$ if $x \in\left[0, \frac{P_{\beta}}{2}\right], \beta(x)=\beta\left(3 P_{\beta}-x\right)$ if $x \in\left[\frac{3 P_{\beta}}{2}, 2 P_{\beta}\right]$.

Thus the trapping and nontrapping terms all disappear, so

$$
a_{0}\left(\phi_{-}\right)=\int_{0}^{2 P_{\beta}}\left|\partial_{x} \phi_{-}\right|^{2}-\int_{\mathbf{R}} \mu^{\prime}(e) d v\left|\phi_{-}\right|^{2} d x<0
$$

For $k \geq 3$, we can simply construct $\phi_{-}(x)$ as follows: In $\left[0,2 P_{\beta}\right]$, it is just the above function. In $\left[2 P_{\beta}, k P_{\beta}\right]$, we merely let $\phi_{-}(x) \equiv \phi_{-}(0)$. Then this $\phi_{-}$ is a $k P_{\beta}$ - periodic function in $H^{1}\left(0, k P_{\beta}\right)$. And it is easy to see that (12) again holds.

\section{Acknowledgement}

The author thanks his advisor, Walter Strauss for continuous encouragement, patience and so many stimulating discussions. Without his help, this work would be impossible. He also thanks Yan Guo for many helpful discussions.

\section{References}

[1] Bernstein, I., Greene, J., Kruskal, M., Exact nonlinear plasma oscillations. Phys. Rev. 108, 3, 546-550 (1957).

[2] Armstrong, T., Montgomery, D., Asymptotic state of the two-stream instability, J.Plasma. Physics, 1, part 4, 425-433 (1967).

[3] Goldman, M. V., Theory of stability of large periodic plasma waves, Phys, Fluids, 13, no. 3, 1281-1289 (1970).

[4] Schwarzmeier, J. L., Lewis, H. R., Abraham-Schrauner, B., Symon, K. R., Stability of Bernstein-Greene-Kruskal equilibria, Phys, Fluids, 22, no. 9, 1747-1760 (1979).

[5] Gizzo, A., Izrar, B., Bertrand, P., Fijalkow, E., Feix, M. R., Shoucri, M., Stability of Bernstein-Greene-Kruskal plasma equilibria. Numerical experiments over a long time, Phys, Fluids, 31, no. 1, 72-82 (1988).

[6] Manfredi, G., Bertrand, P., Stability of Bernstein-Greene-Kruskal modes, Phys. Plasmas, 7, 6, 2425-2431 (2000). 
[7] Grillakis, M., Linearized instability for nonlinear Schrödinger and Klein-Gordon equations. Comm. Pure Appl. Math. 41, no. 6, 747-774 (1988).

[8] Guo, Y., Stable magnetic equilibria in collisionless plasma, Comm. Pure Appl. Math., Vol L, 0891-0933 (1997).

[9] Guo, Y., Stable magnetic equilibria in a symmetric plasma, Commun. Math. Phys., 200, 211-247 (1999).

[10] Guo, Y., Strauss, W., Instability of periodic BGK equilibria, Comm. Pure Appl. Math. Vol XLVIII, 861-894 (1995).

[11] Guo, Y., Strauss, W., Unstable relativistic BGK waves, Comp. and Appl. Math., 18, no. 1, 87-122 (1999).

[12] Guo, Y., Strauss, W., Unstable BGK solitary waves and collisionless shocks, Commun. Math. Phys. 195, 249-265 (1998).

[13] Guo, Y., Strauss, W., Unstable oscillatory-tail solutions, SIAM J. Math. Anal., 30, no. 5, 1076-1114 (1999).

[14] Guo, Y., Strauss, W., Magnetically created instability in a collisionless plasma, J. Math. Pures. Appl., 79, no. 10, 975-1009 (2000).

[15] Golse, F., Lions, P. L., Perthame, B., and Sentis, R., Regularity of the moments of the solution of a transport equation, J. Funct. Anal., 76, no. 1, 110-125 (1988).

[16] DiPerna, R. J., Lions, P. L., Global weak solutions of Vlasov-Maxwell systems, Comm. Pure Appl. Math., Vol XLII, 729-757 (1989).

[17] Reed, M., Simon, B., Methods of modern mathematical physics, Vol IV, Academic press 1978.

[18] Kato, T., Perturbation theory for linear operators, Springer 1976.

Department of Mathematics, Brown University, Providence, RI,02912

E-mail address: linzw@math.brown.edu 